\section{Equivalence of Mass and Energy}

THE following describes an ideal experiment from which the law of equivalence of mass and energy may be derived by using means employed in elementary physics.

Suppose a cylindrical tube entirely evacuated at the ends of which mirrors $\mu_{1}$ and $\mu_{2}$ are placed perpendicular to the generating line of the cylinder, and let the coefficient of absorption of $\mu_{1}$ and $\mu_{2}$ be zero. A ray of light perpendicular to the mirrors will be therefore reflected from $\mu_{1}$ and $\mu_{2}$ for an indefinitely long time.

Consider a monochromatic ray composed of photons, the mass of each photon being $m$ and the velocity of each being $c$ (in vacuum). The impulse of each photon is therefore equal to $m c$, and after reflexion it reverses its direction only, if we assume that photons have absolute elasticity (an assumption made, for example, in the Compton effect).

Applying the method used in the elementary kinetic theory of gases, we get

$$
p=2 m c \cdot N \cdot \frac{c}{2 a} \cdot \frac{1}{S},
$$

where $p$ signifies the mean pressure of a bundle of parallel rays on on $\theta$ of the mirrors, $N$ is the total number of photons in the tube, $a$ is the length of the tube, and $S$ is its area of cross-section.

Substituting the volume $V$ for $a S$,

$$
p V=m c^{2} N \text {. }
$$

An infinitesimal parallel displacement of one mirror towards the other will diminish the volume of the tube by $d V$. The amount of work done by this displacement is $p d V$. Evidently, this amount is equal to the energy increase of the 'photonic gas', or of all its $N$ photons together.

Differentiation of the preceding formula gives

$$
p \cdot d V=c^{2} N \cdot d m,
$$

since the number of photons does not change and the velocity $c$ (according to a postulate of the theory of relativity) remains unchanged too.

Indicating by $d E$ the energy increase of each photon, we get

$$
\frac{p d V}{N}=d E=c^{2} d m \text {. }
$$

Moving the mirrors towards each other by a finite distance and integrating, we get

$$
E-E_{0}=c^{2}\left(m-m_{0}\right) ;
$$

or

$$
E-m c^{2}=E_{0}-m_{0} c^{2}=\text { constant, }
$$

for any pair of values of $E$ and $m$.

Hence

$$
E=m c^{2}+\text { constant. }
$$

Since an expression of energy leaves an additive constant free, we may so choose the latter that

$$
E=m c^{2} \text {. }
$$

The above proof of this fundamental formula applies, of course, only to the special case of the energy of photons.

Hebrew Technical College,

A. TCherniavsky

Haifa.

\section{An Azimuthal Method of Measuring Cloud Height with a Searchlight}

THE normal method of determining the height of the cloud base at night is to direct a vertical searchlight beam on to the cloud and measure the angle of elevation $E$ of the spot of light from a point at a known horizontal distance $L$ from the searchlight. The height $h$ of the cloud base is then given by $h=L \tan E$. The length $L$ of the base-line is normally about $1,000 \mathrm{ft}$

The precision of the method clearly depends upon the accuracy with which the angle of elevation $E$ can be measured. In practice, a limit to the observational accuracy is set by the fact that the spot of light is never sharply defined, because of the diffuse nature of the cloud surface. A reading to the nearest whole degree is therefore the best that can be expected.

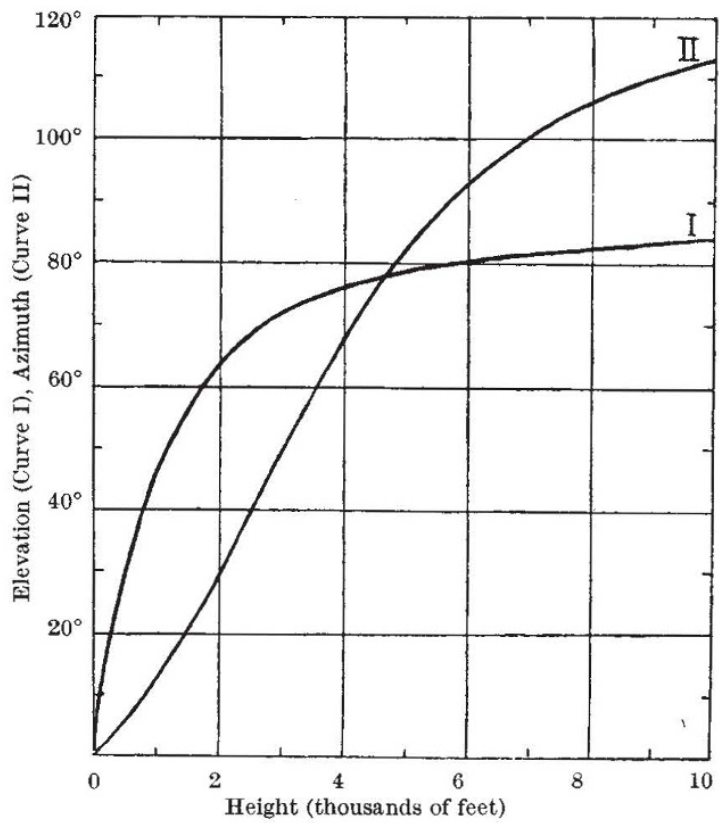

CURVE I: VARIATION OF ELEVATION WITH HEIGHT OF ClOUD; VERTICAL BEAM ; BASE-LINE, 1,000 FT. CURVE II : VARIATION VERTICAL BEAM; BASE-LINE, 1,000 FT. CURVE II: VARIATION LINE, 1,000 FT. ; DIRECTION OF BEAM, $45^{\circ}$ FROM DIRECTION OF BASE-LINE

Curve I shows the relationship between $E$ and $h$ for the case $L=1,000 \mathrm{ft}$. It will be seen that for heights exceeding $4,000 \mathrm{ft}$. the rate of increase of $E$ with $h$ is very small. The increase of elevation between $h=5,000 \mathrm{ft}$. and $h=10,000 \mathrm{ft}$. is, in fact, only $5 \cdot 6^{\circ}$. As $E$ can only be measured to the nearest degree, cloud heights in this range can therefore only be measured to about the nearest 1,000 ft. The new code for height of cloud adopted by the International Meteorological Committee at Paris in 1946 provides for reporting observations to the nearest $300 \mathrm{ft}$. in this range. It is necessary, therefore, to consider means of improving upon the accuracy obtainable by the present method.

If the searchlight beam is tilted out of the vertical in a direction transverse to the line of sight, both the azimuth and elevation of the spot of light will vary with the height of the cloud. It is possible, therefore, to make use of the variation of azimuth to determine the height of the cloud. If $\alpha$ is the angle of inclination of the beam to the horizontal plane, $\beta$ is the angle of orientation of the beam and $\theta$ is the 